\title{
Quando introduzir o tratamento farmacológico na pré-eclâmpsia
}

\author{
Saulo D. da Silva, ${ }^{1}$ Barbara A. Louback, ${ }^{1}$ Denise L. M. Monteiro, ${ }^{1,2^{\star}}$ Alexandre J. B.Trajano, ${ }^{2,3}$ Nilson R. de Jesús ${ }^{2}$
}

\section{Resumo}

Sabe-se que cerca de $10 \%$ das gestações cursam com hipertensão arterial, o que representa a primeira causa de mortalidade materna no Brasil. A pré-eclâmpsia é condição sistêmica que se manifesta clinicamente com o aparecimento de hipertensão arterial após a $20^{\mathrm{a}}$ semana de gestação, em mulheres previamente normotensas, associada à proteinúria. Não há consenso na literatura sobre a conduta farmacológica mais adequada e tampouco se há vantagens em usar fármacos anti-hipertensivos nos casos de pré-eclâmpsia. Sendo assim, este artigo busca reunir informações de pesquisa em bancos de dados, como Cochrane Library, Medline (por meio do PubMed), Google Acadêmico e SciELO, a fim de revisar a conduta proposta atualmente para o tratamento antihipertensivo de manutenção da pré-eclâmpsia. As mais usadas são aquelas que não trazem riscos ao binômio maternofetal ou seus riscos são mínimos, como a hidralazina, nifedipina, alfametildopa e labetalol. Contudo, concluímos que não há conduta-padrão e, sendo assim, a escolha do medicamento vai depender da experiência de cada obstetra e da rotina adotada para aquele serviço.

Descritores: Pré-eclâmpsia; Anti-hipertensivos; Gravidez.

\section{Abstract \\ When to start pharmacological treatment in pre- eclampsia}

It is known that about $10 \%$ of pregnancies occur under hypertensive disorders, representing the main cause of maternal mortality in Brazil. Preeclampsia, systemic condition that is manifested clinically with the onset of hypertension after the twentieth week of gestation in previously normotensive women, associated with proteinuria. There is no consensus regarding the most appropriate pharmacological management or if there is any advantage in using drug treatment in hypertensive situations of mild preeclampsia. Therefore, this article aims to gather research information in databases such as Cochrane Library, Medline (via PubMed), Google Scholar and SciELO, in order to review current maintenance treatment proposed for preeclampsia. The most commonly used are those that do not pose risks to both mother and fetus and its risks are minimal, such as hydralazine, nifedipine, alpha-methyldopa and labetalol. Although, we concluded that there is no standard procedure and, therefore, the choice of drug will depend on the experience of each obstetrician and the routine adopted in that service.

Keywords: Preeclampsia; Antihypertensiveagents; Pregnancy.
1. Departamento de Ginecologia e Obstetrícia. Centro Universitário Serra dos Órgãos. Teresópolis, RJ, Brasil.

2. Departamento de Ginecologia e Obstetrícia. Faculdade de Ciências Médicas. Universidade do Estado do Rio de Janeiro. Rio de Janeiro, RJ, Brasil.

3. Departamento de Ginecologia e Obstetrícia. Escola de Ciências da Saúde. Universidade do Grande Rio. Rio de Janeiro, RJ, Brasil.

\author{
*Endereço para correspondência: \\ Núcleo Perinatal, HUPE, UERJ \\ Av. Prof. Manuel de Abreu, 500, 10 andar \\ Rio de Janeiro, RJ, Brasil. CEP: 20550-170. \\ E-mail: denimonteiro2@yahoo.com.br
}

Revista HUPE, Rio de Janeiro, 2015;14(2):87-93

doi: 10.12957/rhupe.2015.19240

Recebido em 18/09/2014. Aprovado em 14/01/2015.

\section{Resumen}

\section{Cuando introducir el tratamiento farmacológico en la preeclampsia}

Se sabe que aproximadamente el 10\% de los embarazos transcurren con hipertensión arterial, lo que representa la primera causa de mortalidad materna en el Brasil. La preeclampsia es una enfermedad sistémica que se manifiesta clínicamente con la aparición de la hipertensión arterial después de la 20 semana de embarazo, en mujeres previamente normotensas, asociada con la proteinuria. No hay consenso en la literatura sobre la conducta farmacológica más adecuada y tampoco si hay ventajas de usar fármacos antihipertensivos en los casos de preeclampsia. Por lo tanto, este artículo busca reunir informaciones sobre la investigación de las bases de datos, como Cochrane Library, Medline (por medio del PubMed), Google Académico y SciELO con el fin de reconsiderar la conducta actualmente propuesta para el tratamiento antihipertensivo de mantenimiento de la preeclampsia. Los más utilizados son los que no plantean riesgos para el binomio maternofetal o sus riesgos son mínimos, como la hidralazina, nifedipina, alfa-metildopa y labetalol. Sin embargo, se concluye que no existe un padrón de conducta y, por lo tanto, la elección del medicamento dependerá de la experiencia de cada obstetra y de la rutina adoptada para aquel servicio.

Palabras clave: Preeclampsia; Anti-hipertensivos; Embarazo. 


\section{Artigo de revisão}

\section{Introdução}

Na fisiologia da gestação normal, ocorre aumento do volume de sangue circulante e concomitantemente, declínio da resistência vascular periférica, com redução da pressão arterial (PA) nas primeiras semanas de gravidez. A este processo fisiológico, segue-se o retorno gradual da $\mathrm{PA}$, atingindo os valores pré-gravídicos próximo ao termo. ${ }^{1}$ Entretanto, aproximadamente $10 \%$ das gestações cursam com hipertensão arterial, especialmente em países em desenvolvimento, representando a primeira causa de mortalidade materna no Brasil. ${ }^{2} \mathrm{~A}$ hipertensão arterial não controlada aumenta o risco materno de insuficiência cardíaca, eventos vasculares cerebrais e insuficiência renal. Pode também causar danos ao concepto como prematuridade, crescimento intrauterino restrito, baixo peso ao nascer e morte fetal intrauterina. $^{3}$

As síndromes hipertensivas na gestação podem ser classificadas em hipertensão gestacional (PA $\geq 140$ x 90 $\mathrm{mmHg}$, diagnosticada pela primeira vez na gestação, ausência de proteinúria, retorno aos níveis tensionais até 12 semanas após o parto); hipertensão arterial crônica (PA $\geq 140$ x 90 mmHg prévia à gestação ou antes de 20 semanas de gestação e que persiste após 12 semanas do parto); pré-eclâmpsia ( $\mathrm{PA} \geq 140$ x 90 mmHg diagnosticada após 20 semanas de gestação associada à proteinúria > $300 \mathrm{mg} / 24$ horas); pré-eclâmpsia sobreposta (surgimento de proteinúria $\geq 300 \mathrm{mg} / 24$ horas em paciente hipertensa que não apresentava proteinúria antes de 20 semanas de gestação ou aumento importante da proteinúria, da pressão arterial ou plaquetas < $100.000 / \mathrm{mm}^{3} \mathrm{em}$ gestante hipertensa com proteinúria presente antes de 20 semanas de gestação). A eclâmpsia é a complicação definida com a presença de convulsão em gestante com pré-eclâmpsia. ${ }^{4}$

Conceitua-se pré-eclâmpsia ( $\mathrm{PE}$ ) como desordem multissistêmica que se manifesta clinicamente com o aparecimento de hipertensão arterial em algum momento após a $20^{\mathrm{a}}$ semana de gestação, em mulheres previamente normotensas, associada à proteinúria maior ou igual a $300 \mathrm{mg}$ em urina de 24 horas ou $1+$ em fita reativa. ${ }^{1,3}$

Para o diagnóstico, a PA deve ser aferida com a gestante sentada e com o braço ao nível do coração, e a fase V de Korotkoff deve ser usada para designar a

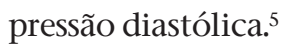

A International Society for the Study of Hypertension in Pregnancy define a hipertensão como a PA diastólica maior ou igual a 90mmHg em aferições separadas em intervalo de quatro horas, ${ }^{1}$ e a The Society of Obstetrician and Gynecologists of Canada (SOGC) recomenda em seu consenso que a PA sistólica seja excluída da definição de hipertensão na gestação, e que esta seja definida com PA diastólica maior ou igual a $90 \mathrm{mmHg}$ em duas aferições, sendo que, mulheres com PA sistólica maior ou igual a $140 \mathrm{mmHg}$ devem ser acompanhadas atentamente para o desenvolvimento de hipertensão diastólica. ${ }^{6}$

O tratamento da PE leve (PA $\geq 140$ x 90 e $<160$ x 110) se baseia em três aspectos principais: a cuidadosa avaliação clínica e complementar da mãe e do concepto; poucas intervenções medicamentosas ou dietéticas e cuidadoso planejamento da conduta obstétrica. No Núcleo Perinatal HUPE-UERJ são recomendadas as seguintes providências:

a) programar assistência pré-natal com controle mais frequente. Além das consultas, deve-se programar a avaliação do peso e da PA, se possível, duas vezes por semana;

b) suspeita de PE, caracterizada por aumento da PA, porém ainda abaixo de 140 x 90 ou ganho ponderal excessivo (> 1 a $2 \mathrm{~kg} / \mathrm{sem}$ ), já deve indicar consultas mais frequentes, com vista ao diagnóstico precoce da PE;

c) idealmente, a paciente deve ser internada por alguns dias para avaliação mais precisa do quadro e decisão se deverá ser mantida internada (ex.: PA diastólica maior ou igual a $100 \mathrm{mmHg}$ após cuidados iniciais ou suspeita de comprometimento fetal) ou acompanhada ambulatorialmente (ex.: PA diastólica sempre menor que $100 \mathrm{mmHg}$ sem qualquer comprometimento fetal);

d) é recomendado repouso relativo (se possível, duas horas durante o dia, em decúbito lateral).

A pré-eclâmpsia grave é definida como aumento da PA para valores maiores que $160 / 110 \mathrm{mmHg}$ e maior ou igual a $2 \mathrm{~g}$ de proteína em urina de 24 horas, ${ }^{1}$ apresentando pior prognóstico maternofetal. ${ }^{4}$ Outros indicativos de pré-eclâmpsia grave são a elevação das enzimas hepáticas, distúrbios visuais e cerebrais, cefaleia severa, oligúria ( $\leq 20 \mathrm{ml} /$ hora), dor epigástrica ou no quadrante superior direito, trombocitopenia e hemólise, que caracterizam a síndrome HELLP. ${ }^{7}$

Muitos mecanismos têm sido propostos para explicar a etiologia da pré-eclâmpsia, envolvendo fatores maternos, placentários e fetais. Entre eles, os considerados mais importantes envolvem a invasão trofoblástica anormal e não adaptação dos vasos uterinos para a gestação, tendo como consequência a redução do fluxo 
sanguíneo placentário e fetal, diminuição da perfusão e isquemia, o que levaria à disfunção endotelial e desequilíbrio de fatores vasodilatadores e vasoconstritores, resultando em grande aumento da resistência vascular periférica e hipertensão arterial., ${ }^{5,8}$

Em função de não haver consenso sobre ouso de tratamento anti-hipertensivo na pré-eclâmpsia, esta revisão tem como objetivo avaliar a efetividade e a segurança do tratamento farmacológico da pré-eclâmpsia para o binômio maternofetal, assim como responder a questionamentos sobre quando começar a terapêutica, levando em consideração os graus de recomendação dos anti-hipertensivos usados no Brasil e em outros países do mundo.

\section{Métodos}

Esta revisão crítica da literatura procura apresentar as drogas relacionadas com o tratamento farmacológico anti-hipertensivo da pré-eclâmpsia. Para isso, foi realizada ampla pesquisa na literatura disponível nos seguintes bancos de dados, na língua inglesa e portuguesa: Medline/PubMed, SciELO e biblioteca Cochrane. Para a pesquisa no Medline, foi utilizada a seguinte estratégia de busca: ("Antihypertensive Agents "[Mesh]) and "Pre-Eclampsia/therapy" [Mesh]), nos últimos 10 anos, sendo encontrados 153 artigos. Para as outras bases, foram usadas as palavras-chave: hipertensão, gravidez, pré-eclâmpsia, antihypertensive drugs and therapy. A busca resultou em 162 artigos que foram avaliados por dois revisores, sendo selecionados 31 estudos para desenvolver este trabalho, obedecendo aos critérios de inclusão e exclusão pré-estabelecidos (Figura 1e Tabela 1).

Para a classificação dos trabalhos citados na revisão,

Figura 1. Fluxograma da pesquisa bibliográfica e seleção dos artigos.

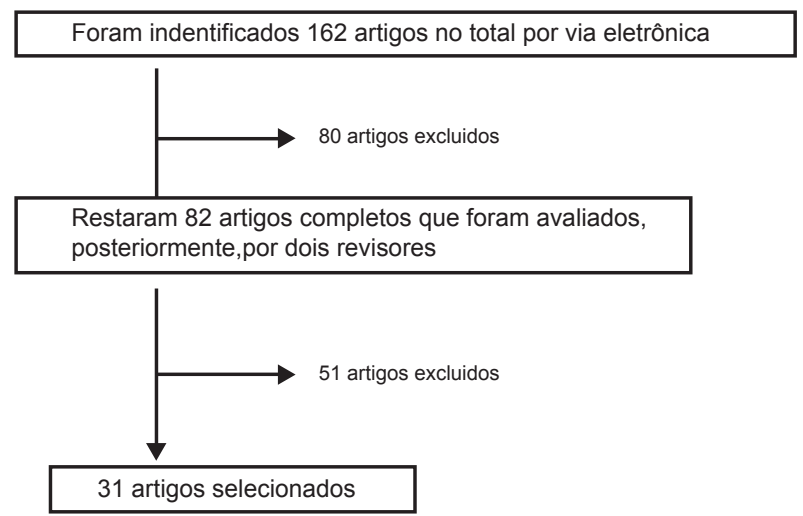

utilizou-se a classificação proposta pela Associação Médica Brasileira (Tabela 2).

\section{Resultados e discussão}

Geralmente a pré-eclâmpsia se resolve com o parto, que permanece como o tratamento definitivo (B). ${ }^{9}$ Apesar de ser pouco descrita na literatura, existe a possibilidade de ocorrência da pré-eclâmpsia no puerpério, com taxas de prevalência variando de $0,3 \%$ a $27,5 \%$, de acordo com recente revisão (D)..$^{10}$

Não está claro o papel que a terapia farmacológica anti-hipertensiva cumpre em mulheres com hipertensão leve a moderada, e seus benefícios não têm sido demonstrados em ensaios clínicos (A). ${ }^{1}$ Sabe-se que mesmo ocorrendo vasodilatação da artéria uterina, não há diminuição da resistência da circulação placentária (B). ${ }^{11}$

O principal objetivo do tratamento da pré-eclâmpsia é o bem-estar materno, ${ }^{7}$ pois previne hemorragia intracerebral e falência cardíaca sem afetar a perfusão cerebral e o fluxo sanguíneo uteroplacentário que já está reduzido na gestante com pré-eclâmpsia. ${ }^{12} \mathrm{~A}$

\section{Tabela 1. Critérios de inclusão e exclusão.}

\begin{tabular}{|c|c|}
\hline Critérios de inclusão & Critérios de exclusão \\
\hline $\begin{array}{l}\text { 1. Foco no tratamen- } \\
\text { to anti-hipertensivo da } \\
\text { pré-eclâmpsia. }\end{array}$ & $\begin{array}{l}\text { 1. Foco no tratamento da } \\
\text { hipertensão crônica na } \\
\text { gestação. }\end{array}$ \\
\hline $\begin{array}{l}\text { 2. Estudos em português, } \\
\text { inglês, espanhol e francês. }\end{array}$ & $\begin{array}{l}\text { 2. Estudos que abordam } \\
\text { tratamento da eclâmpsia. }\end{array}$ \\
\hline 3. Estudos a partir de 2002. & $\begin{array}{l}\text { 3. Estudos sobre trata- } \\
\text { mento para prevenção da } \\
\text { pré-eclâmpsia. }\end{array}$ \\
\hline & $\begin{array}{l}\text { 4. Artigos com texto com- } \\
\text { pleto inacessíveis. }\end{array}$ \\
\hline
\end{tabular}

Tabela 2. Grau de recomendação e força de evidência.

\begin{tabular}{|c|c|}
\hline A & $\begin{array}{l}\text { Estudos experimentais ou observacionais de } \\
\text { melhor consistência (metanálises ou ensaios } \\
\text { clínicos randomizados). }\end{array}$ \\
\hline B & $\begin{array}{l}\text { Estudos experimentais ou observacionais de } \\
\text { menos consistência (outros ensaios clínicos não } \\
\text { randomizados ou estudos observacionais ou } \\
\text { estudos caso-controle). }\end{array}$ \\
\hline C & $\begin{array}{l}\text { Relatos ou séries de casos (estudos não contro- } \\
\text { lados). }\end{array}$ \\
\hline D & $\begin{array}{l}\text { Opinião desprovida de avaliação crítica, baseada } \\
\text { em consensos, estudos fisiológicos ou modelos } \\
\text { animais. }\end{array}$ \\
\hline
\end{tabular}




\section{Artigo de revisão}

terapia anti-hipertensiva não previne a pré-eclâmpsia, a morte neonatal, nascimentos prematuros e nem o crescimento intrauterino restrito, mas diminui pela metade a incidência de hipertensão grave entre gestantes com hipertensão leve., ${ }^{1,5,6,8}$ Revisão sistemática da biblioteca Cochrane incluindo 28 ensaios clínicos com 3.200 mulheres, comparando uso de fármaco com placebo evidenciou que o uso de anti-hipertensivos reduziu o risco de desenvolver hipertensão grave pela metade (RR 0,50 IC 95\% (0,41-0,61)) e esse efeito ocorreu independentemente do tipo de fármaco (A). ${ }^{1}$

Não há unanimidade em relação à indicação do tratamento farmacológico na pré-eclâmpsia. A diretriz de 2008 da The Society of Obstetrician and Gynecologists of Canada (SOGC) recomenda que mulheres com hipertensão leve (PA 140-159/90-109 mmHg) sem comorbidades, devem ser tratadas com hipotensores para manter a PA sistólica entre 130-155 mmHg e PA diastólica entre 80-105 mmHg. Para mulheres com hipertensão grave recomendam que a PA sistólica seja mantida menor ou igual a $160 \mathrm{mmHg}$ e a diastólica menor ou igual a $110 \mathrm{mmHg}$ (D). ${ }^{6}$ Nos Estados Unidos, a terapêutica é recomendada se PA maior ou igual a 160 x 105 mmHg, e na Austrália trata-se quando a PA maior ou igual a 160 x $90 \mathrm{mmHg}$ e recomendam manter a PA sistólica abaixo ou igual a $110 \mathrm{mmHg}$ (D). ${ }^{5}$

O Royal College of Obstetricians and Gynecologists (RCOG) britânico indica tratamento materno quando a PA sistólica for maior ou igual a $160 \mathrm{mmHg}$ ou a diastólica acima ou igual a $110 \mathrm{mmHg} .{ }^{13}$ Pequena proporção de hipotensores disponíveis tem sido avaliada adequadamente em mulheres grávidas, e muitos outros são contraindicados (D). ${ }^{9}$

A The Food and Drug Administration(FDA) classifica a maioria dos anti-hipertensivos usados na gravidez como categoria "C", na qual os estudos em humanos são escassos e estudos realizados em animais são positivos para risco fetal ou estão faltando e, nessa categoria, a droga deve ser dada apenas se os benefícios potenciais justificarem os riscos potenciais para o feto (D). ${ }^{14}$

Sibai ${ }^{9}$ recomenda que o hipotensor seja usado apenas nas pacientes com PE grave. ${ }^{9}$ Esta é a orientação que seguimos no Núcleo Perinatal HUPE-UERJ em que, enquanto a PA se mantiver abaixo de 160 x 110 mmHg, não devem ser usados hipotensores ou diuréticos. Solicitar exames complementares para avaliar a saúde materna ("rotina de hipertensão"), que devem ser repetidos de acordo com a gravidade do caso: hemograma com contagem de plaquetas, proteinúria de 24 horas, dosagem da ureia, creatinina, ácido úrico e enzimas hepáticas.
Na suspeita de hipertensão crônica associada deve-se solicitar fundo de olho (FO) e eletrocardiograma (ECG).

Realizar ultrassonografia (USG) a cada duas a quatro semanas para avaliar o crescimento fetal, que deve estar acima do décimo percentil e avaliar o volume do líquido amniótico pela medida do maior bolsão vertical. Realizar dopplervelocimetria da artéria umbilical e da artéria cerebral média mensalmente nos casos leves e a cada uma ou duas semanas nos casos graves. Havendo comprometimento da dopplervelocimetria arterial, pode-se empregar exames que se alteram mais tardiamente, como a dopplervelocimetria do ducto venoso e a cardiotocografia.

Nos casos de PE grave, o tratamento se baseia em cinco aspectos principais: internar todas as pacientes até o final da gravidez, tratamento emergencial da hipertensão arterial grave (PA $\geq 160$ x 110), prevenção da convulsão, cuidadosa avaliação clínica e complementar da mãe e do concepto, avaliação da necessidade de terapia hipotensora adicional e planejamento da conduta obstétrica.

O primeiro passo da emergência hipertensiva com idade gestacional acima de 34 semanas é a internação hospitalar, seguida do uso de sulfato de magnésio para prevenção de convulsão, além do uso de drogas anti-hipertensivas, ${ }^{15}$ (D) como a hidralazina e labetalol por via intravenosa ou nifedipina por via oral. ${ }^{16}$

A orientação que seguimos no Núcleo Perinatal HUPE-UERJ em todas as pacientes com PE grave é a administração de sulfato de magnésio com dose de ataque: $4 \mathrm{~g} \mathrm{EV}$, administrados lentamente (10 minutos) e dose de manutenção: $2 \mathrm{~g} / \mathrm{h}$ por período entre 24 e 48 horas, em bomba infusora. Medicar com hipotensor, hidralazina ${ }^{5}$ mg EV, a cada 20 minutos, até quatro doses. O objetivo do tratamento é o controle da PA $(<160$ x $110 \mathrm{mmHg})$ e não a sua normalização (<140 x 90 mmHg).

Após a terapia de ataque, se a paciente se manteve com a PA controlada, sempre abaixo de $160 \times 110 \mathrm{mmHg}$, será mantida internada sob rigorosa vigilância. Se a avaliação do bem-estar fetal estiver normal, será indicada indução do parto ou cesárea com 35-37 semanas e se o feto estiver levemente comprometido, deverá ser submetida à cesárea com 35 semanas.

Após a terapia de ataque, se a PA não foi controlada, apresentando valores acima de 160 x 110 mmHg: se a idade gestacional for acima ou igual a 32 semanas, deve-se optar pela interrupção da gravidez. Se a idade gestacional for menor que 32 semanas, pode-se empregar medicação hipotensora por via oral. Caso haja controle da PA com a medicação oral, a gravidez pode ser 
seguida até 32 semanas. Caso não haja controle da PA, deve-se interromper a gravidez, independentemente da idade gestacional.

A seguir estão descritos alguns esquemas terapêuticos para este hipotensor adicional: hidralazina (75-200 $\mathrm{mg} /$ dia), metildopa (750-2.000 mg/dia), nifedipina (20-60 mg/dia), pindolol (5-30 mg/dia) e verapamil (240-480 mg/dia), com o objetivo de manter a pressão sistólica entre 140-155 mmHg e diastólica entre 90-105 mmHg (D). ${ }^{9}$

A metildopa é um alfa-agonista que inibe a vasoconstrição através de efeito mediado a nível central. Por via oral,é o hipotensor mais frequentemente utilizado na gravidez (A). ${ }^{1}$ É também a droga anti-hipertensiva mais bem-estudada, segura e considerada de primeira linha (A). ${ }^{17}$ Ela diminui o espasmo vascular uteroplacentário na artéria uterina, porém não altera a resistência da artéria umbilical nem os parâmetros fetais. ${ }^{18}$

Em oito ensaios envolvendo 493 mulheres, a metildopa pareceu ser menos efetiva que os betabloqueadores para prevenir a hipertensão arterial grave $(\mathrm{RR}=$ 0,79; IC 95\% 0,63-0,99), contudo os efeitos comparativos sobre outras medidas de resultados são incertos (A). ${ }^{1}$

A hidralazina é potente vasodilatador que reduz a resistência vascular periférica, relaxando diretamente o músculo liso dos vasos arteriais, ${ }^{11}$ sendo uma das drogas de escolha nas emergências hipertensivas gestacionais (A). ${ }^{1}$ Esse fármaco pode causar efeitos colaterais maternos em função do seu efeito farmacológico incluindo taquicardia, cefaleia, hipotensão, náusea, palpitação, tonteira e retenção de sal (A,B). ${ }^{19,20}$

A nifedipina é bloqueador dos canais de cálcio tipo 2. Essa ação leva primariamente ao relaxamento do músculo liso, atuando principalmente sobre a musculatura vascular, uterina e vesical. ${ }^{21}$ Esta droga inicia sua ação na gravidez, aproximadamente, em dez a 30 minutos. O pico de redução da pressão arterial ocorre com 40 a 75 minutos. A duração de sua ação é variável de duas a quatro horas, mas pode persistir ao longo de oitro horas (B). ${ }^{22}$ Suas principais aplicações na gestação são para o tratamento da emergência hipertensiva, no tratamento da hipertensão por tempo prolongado e também como agente tocolítico (A). ${ }^{23}$

Recente metanálise de 21 estudos concluiu que o labetalol parenteral ou a nifedipina oral foram preferíveis como agentes de primeira linha e a hidralazina, um agente conveniente de segunda linha. Comparativamente a outros fármacos, as pacientes tratadas com hidralazina apresentaram com maior frequência hipotensão, oligúria, partos cesáreos e placenta prévia.
Ocorreram mais baixos escores de Apgar e mais efeitos adversos na frequência cardíaca fetal. Entretanto tais dados não foram suficientes para fazer uma recomendação definitiva de uma droga sobre a outra (A). ${ }^{24}$

Os efeitos adversos dos bloqueadores do canal de cálcio incluem edema periférico, cefaleia, taquicardia, palpitação e rubor facial. A nifedipina não parece causar decréscimo detectável do fluxo sanguíneo uterino e quando administrada por via oral no primeiro trimestre, não parece causar efeitos teratogênicos ao feto. Administrada em forma de cápsulas de curta duração, a nifedipina tem sido associada, em alguns casos, com hipotensão materna e sofrimento fetal, contudo já foi demonstrada a eficácia e segurança da nifedipina oral de ação prolongada na hipertensão grave durante a gravidez e, por tudo isso, recomenda-se o uso de preparações de ação prolongada (B). ${ }^{6}$

Ensaio clínico randomizado com 126 pacientes portadoras de pré-eclâmpsia em que 65 pacientes receberam nifepidina e 61 usaram hidralazina, constatou que menores doses de nifedipina foram necessárias para alcançar o controle efetivo da PA quando comparado à hidralazina $(\mathrm{p}=0,03)$, e o intervalo de tempo antes de nova crise hipertensiva, após a estabilização inicial da PA, foi significativamente mais longo no grupo que usou a nifedipina quando comparado ao grupo da hidralazina. Efeitos adversos ocorreram em 16,9\% do grupo da nifedipina e em 16,4\% da hidralazina. Taquicardia foi mais observada com a hidralazina, entretanto a cefaleia foi mais comum nas que usaram nifedipina. Não se observou queda brusca da pressão arterial e as escalas de Apgar dos recém-nascidos não foram significativamente diferentes entre os dois grupos. Em contraste com a hidralazina, a nifedipina é, teoricamente, a candidata ideal para uso na pré-eclâmpsia, por diminuir a PA e poder ser administrada por via oral, enquanto a hidralazina deve ser utilizada por via parenteral (A). ${ }^{25}$

Em revisão sistemática da Cochrane sobre o tratamento anti-hipertensivo de emergência, que incluiu 24 ensaios clínicos randomizados envolvendo 12 comparações, constatou-se menor risco de hipertensão persistente com os bloqueadores dos canais de cálcio (como a nifedipina) em relação à hidralazina (RR: 0,33; IC95\% 0,15-0,70). Porém, concluiu-se que não há dados suficientes para atestar a superioridade de uma droga anti-hipertensiva de emergência em relação à outra na gestação (A). ${ }^{26}$

Os betabloqueadores são drogas anti-hipertensivas que exercem seus efeitos através do bloqueio dos receptores beta 1 e beta 2 , reduzindo a frequência cardíaca, 


\section{Artigo de revisão}

pressão sanguínea, o consumo de oxigênio pelo miocárdio e a contratibilidade do músculo cardíaco (D) ${ }^{27} \mathrm{O}$ labetalol é um competidor $\beta$-adrenérgico não seletivo e competidor $\alpha-1$ adrenérgico seletivo. ${ }^{5}$ Ele reduz a resistência vascular periférica sem causar redução do fluxo sanguíneo periférico total e, além disso, o fluxo cerebral, renal, coronariano e uterino são preservados. A transferência placentária do fármaco élimitada devido à sua baixa solubilidade lipídica (D) ${ }^{28}$

A American Heart Association, a Associação Americana do Coração, recomenda tanto o labetalol quanto a metildopa como agentes de primeira linha para o tratamento da hipertensão não severa durante a gestação. ${ }^{5}$ No NICE Guidelines 2010, o manual desenvolvido pelo Instituto Nacional de Saúde e Excelência em Assistência dos EUA, o labetalol oral foi incluído na lista de anti-HAS como agente de primeira escolha para o tratamento da HAS severa (D). ${ }^{29}$

Os inibidores da enzima conversora da angiotensina (IECA) e os bloqueadores dos receptores da angiotensina II podem agravar a isquemia uterina e causar insuficiência renal no feto, por isso são contraindicados durante a gestação (D). ${ }^{9}$

Segundo a SOGC, ${ }^{6}$ o tratamento inicial da hipertensão leve a moderada pode ser feito com metildopa, labetalol, outros betabloqueadores (acebutolol, metoprolol, pindolol e propranolol) e bloqueadores do canal de cálcio (nifedipina) (C). O atenolol não é recomendado e os IECA não devem ser usados. Para a hipertensão grave, recomenda-se que a terapêutica inicial seja com labetalol, nifedipina ou hidralazina (D). ${ }^{6}$

A RCOG cita que a metildopa e labetalol foram os anti-hipertensivos mais comumente usados no Reino
Unido. Os IECA são contraindicados e recomenda-se que os diuréticos só devam ser usados em casos de edema pulmonar (D). ${ }^{13}$ Ainda segundo a RCOG, ${ }^{13} \mathrm{O}$ atenolol está associado ao aumento de restrição do crescimento fetal, devendo-se evitá-lo. Para a hipertensão grave aguda, também é recomendado o uso de labetalol, nifedipina ou hidralazina (D). ${ }^{10}$

As doses das drogas mais utilizadas para prolongar a gestação nos casos de pré-eclâmpsia estão descritas na tabela 3.

\section{Conclusão}

O controle farmacológico dos níveis pressóricos em gestantes com pré-eclâmpsia ainda não é completamente compreendido e, mesmo que alguns medicamentos possam apresentar eficácia, provocam reações adversas indesejáveis, influenciando na escolha terapêutica.

É notável a diferenciação da grávida como paciente, quando classes de medicamentos consagrados no tratamento da hipertensão arterial sistêmica, como os diuréticos e os IECA são francamente abolidos e contraindicados pela FDA no tratamento da DHEG, assim como também o propranolol e outros representantes dos betabloqueadores, excetuando o labetalol e o pindolol que ainda podem ser tolerados.

Dentre esses medicamentos, o agente antihipertensivo de ação central, a $\alpha$-metildopa é o único medicamento a possuir grau de recomendação B pela FDA e, assim, comumente receitado. Entretanto, a terapêutica deve ser utilizada com cautela considerando todos os efeitos sobre o binômio mãe-feto.

Outros medicamentos utilizados no tratamento

Tabela 3. Fármacos anti-hipertensivos utilizados na gestação.

\begin{tabular}{|c|c|c|c|c|}
\hline Medicamentos & Classe & Via & Dose & FDA \\
\hline$\alpha$-metildopa & Inibidor $\alpha$-adrenérgico & VO & 750 a $2.000 \mathrm{mg} / \mathrm{dia}$ ( 2 a 4 doses) & B \\
\hline Propranolol & $\beta$-bloqueadores & VO & 40 a $160 \mathrm{mg} / \mathrm{dia}$ (1 a 3 doses) & C \\
\hline Nifedipina & Bloqueadordos canais de cálcio & VO & 20 a $60 \mathrm{mg} /$ dia (3 doses) & C \\
\hline Furosemida* & Diurético de alça & VO & Variável & C \\
\hline Hidralazina & Vasodilatador & $\begin{array}{l}\text { VO } \\
\text { EV }\end{array}$ & $\begin{array}{l}75 \text { a } 200 \text { mg/dia ( } 3 \text { a } 4 \text { doses) } \\
5 \text { mg em bólus, repetir em } 30 \text { minutos }\end{array}$ & $\begin{array}{l}\text { C } \\
\text { C }\end{array}$ \\
\hline
\end{tabular}


agudo da hipertensão arterial, como a hidralazina, também aparecem como alternativa, apesar de suas contraindicações. É muito utilizada por via intravenosa nas emergências hipertensivas por ser potente vasodilatador que atua diretamente sobre a musculatura lisa arteriolar.

Diante desse cenário, a conduta ideal na préeclâmpsia é a interrupção da gestação quando há viabilidade fetal. Caso isso não seja possível e havendo hipertensão grave, pode-se lançar mão de drogas antihipertensivas a fim de prolongar a gestação até que o parto seja viável. No entanto, a escolha do anti-hipertensivo ainda desperta questionamentos em relação ao risco-benefício de cada droga, sendo necessários mais ensaios clínicos randomizados na busca incessante pela melhor escolha terapêutica.

\section{Referências}

1. Abalos E, Duley L, Steyn DW, et al. Antihypertensive drugs therapy for mild to moderate hypertension during pregnancy. (Cochrane Review). The Cochrane Library. Issue 2, 2008. Oxford: Update Software.

2. Souza A, Amorim M, Costa A. Efeitos da nifedipina no tratamento anti-hipertensivo da pré-eclâmpsia. Acta Med Port 2008; 21(4):351-8.

3. McCoy S, Baldwin K. Pharmaco therapeutic options for the treatment of preeclampsia. Clinical Review. Am J Health Syst Pharm. 2009; 66(4):337-44.doi: 10.2146/ajhp080104.

4. Oliveira CA, Lins CP, Sá RAM, et al. Síndromes hipertensivas da gestação e repercussões perinatais. Rev Bras Saúde Matern Infant. 2008;6(1):93-8.

5. Podymow T, August P. Update on the use of antihypertensive drugs in pregnancy. Hypertension. 2008; 51(4):960-9. doi: 10.1161/HYPERTENSIONAHA.106.075895.

6. SOGC. Diagnosis, evaluation and managment of the hypertensive disorders of pregnancy.J Obstet Gynaecol Canad. 2008; 30(3Suppl):S1-48.

7. Huarte Ciganda M, Modroño A, Larrañaga C. Management of hypertension in pregnancy.An Sist Sanit Navar. 2009;32 Suppl 1:91-103.

8. Ferrão MH, Pereira AC, Gersgorin HC, Paula TA, Corrêa RR, Castro EC. Treatment effectiveness of hypertension during pregnancy.Rev Assoc Med Bras. 2006; 52(6):390-4.

9. Sibai BM, Barton JR. Expectant management of severe preeclampsia remote from term: patient selection, treatment, and delivery indications. Am J Obstet Gynecol. 2007; 196(6):514.e1-9.

10. Sibai BM. Etiology and management of postpartum hypertension-preeclampsia. Am J Obstet Gynecol. 2012; 206(6):470-5. doi: 10.1016/j.ajog.2011.09.002.

11. Günenç $O$, Çiçek N, Görkemli $H$, et al.The effect of methyldopa treatment on uterine, umblical and fetal middle cerebral artery blood flows in preeclamptic patients. Arch Gynecol Obstet. 2002; 266(3):141-4

12. Sibai BM. Diagnosis, prevention and management of eclamp- sia. Obstet Gynecol 2005; 105(2):401-10.

13. RCOG. The management of severe pre-eclampsia/eclâmpsia. Guideline $n^{\circ}$ 10. 2006. p.1-11.

14. CEMD-UK 2004 National Institute of Clinical Excellence, Scottish Executive Health Department, Department of Health Social Services and Public Safety Northern Ireland. Why mothers die 2000-2002. The sixth report on confidential enquiries into maternal deaths in the United Kingdom.London: RCOG Press, 2004.

15. Lindheimer MD, Barron WM. Medical Disorders in Pregnancy,3rd ed.St. Louis: Mosby; 2000.

16. Chobanian AV, Bakris GL, Black HR, et al. The Seventh Report of the Joint National Committee on Prevention, Detection, Evaluation, and Treatment of High Blood Pressure: the JNC 7 report. JAMA. 2003; 289(19):2560-72.

17. Elhassan EM, Mirghani OA, Habour AB, et al. Methyldopa versus no drug treatment in the management of mild pre-eclampsia. East Afr Med J. 2002; 79(4):172-5

18. Arce C, Segura-Pacheco B, Perez-Cardenas E, et al. Hydralazine target: from blood vessels to the epigenome. Transl Med. 2006; 4:10. doi:10.1186/1479-5876-4-10

19. Jounela AJ, Pasanan M, Matilla MJ. Acetylator phenotype and the antihypertensive response to hydralazine. Acta Med Scand 1975; 197(4):303-6.

20. Johnston GD. Dose-response relationship with antihypertensive drugs. Pharmac Ther 1992; 55(1): 53-93.

21. De Souza AR, Amorim MR, Costa AR. Nifedipine antihypertensive treatment effects in pre-eclampsia. Acta Med Port. 2008;21(4):351-8.

22. Levin AC, Doering PL, Hatton RC. Use of nifedipine in the hypertensive diseases of pregnancy.Ann Pharmacother. 1994;28(12):1371-8.

23. King JF, Flenady VJ, Papatsonis DNM, et al. Calcium channel blockers for inhibiting preterm labour (Cochrane Review). In: The Cochrane Library, Oxford:Update Software. 2007.

24. Magee LA, Cham C, Waterman EJ, et al. Hydralazine for treatment of severe hypertension in pregnancy: meta-analysis. BMJ. 2003; 327(7421):955-60.

25. Aali BS, Nejad SS. Nifedipine or hydralazine as a first-line agent to control hypertension in severe preeclampsia.ActaObstetGynecol Scand. 2002; 81(1):25-30.

26. Duley L, Henderson-Smart DJ. Drugs for treatment of very high blood pressure during pregnancy.Cochrane Database Syst Rev. 2010.

27. Ghanem FA, Movahed A. Use of antihypertensive drugs during pregnancy and lactation.CardiovascTher. 2008; 26(1):38-49. doi: 10.1111/j.1527-3466.2007.00036.x.

28. Pearce CJ, Wallin JD.Labetalol and other agents that block both alpha- and beta-adrenergic receptors. Cleve Clin J Med. 1994; 61(1): 59-69.

29. National Institute for Health and Clinical Excellence. Hypertensive disorders during pregnancy. 2010. Disponível em:http:// guidance.nice.org.uk/CG/Wave15/10. Acesso em 15 Ago 2014. 